\title{
Designing a knowledge management system for Naval Materials Failures
}

\author{
Nikolaos Melanitis ${ }^{1 *}$, George Giannakopoulos ${ }^{2}$, Konstantinos Stamatakis $^{2}$, Dionysios \\ Mouzakis $^{3}$, Aggelos Koutsomichalis ${ }^{4}$ \\ ${ }^{1}$ Hellenic Naval Academy, Piraeus, Greece \\ ${ }^{2}$ Institute of Informatics \& Telecommunications, NCSR Demokritos, Athens, Greece \\ ${ }^{3}$ Hellenic Army Academy, Vari, Greece \\ ${ }^{4}$ Hellenic Air Force Academy, Dekeleia, Greece
}

\begin{abstract}
NAVMAT Research project attempts an interdisciplinary approach by integrating Materials Engineering and Informatics under a platform of Knowledge Management. Failure analysis expands into forensics engineering for it aims not only to identify individual and symptomatic reasons of failure but to assess and understand repetitive failure patterns, which could be related to underlying material faults, design mistakes or maintenance omissions. NAVMAT approach utilizes a focused common-cause failure methodology for the naval and marine environment, to begin with. It will eventually support decision making through appropriate Artificial Intelligence and Natural Language Processing methods. The presented work describes the design of a knowledge based system dedicated to effective recording, efficient indexing, easy and accurate retrieval of information, history of maintenance and secure operation concerning failure incidents of marine materials, components and systems in a fleet organisation. Based on materials failure ontology, utilising artificial intelligence algorithms and modern approaches in data handling, NAVMAT aims at the optimisation of naval materials failure management and the support of decision making in Maintenance and Repair Operations (MRO), materials supplies and staff training.
\end{abstract}

\section{Introduction: addressing the need}

Some of the most important concerns in the management of materials and systems failure for a large organisation (industry, service provider) such as a fleet and/or its technical departments are: the meticulous recording and indexing of an incident, the assessment and identification of causes, the proposal of feasible solutions. To meet these concerns, quick access and retrieval of knowledge is a prerequisite. The related information is recorded at the platform level (ship, building, unit), but its concentration and collection at central level and redistribution as a knowledge and guideline is not an easy process. As a result, problem solving operations may repeat when the same or similar problem appears in another

* Corresponding author: melanitis@hna.gr 
location of the Organisation, human and financial resources are "wasted" for re-interpreting the incident and operational readiness may be compromised. The disruption in the flow of knowledge (knowledge gap) may be intensified taking into account:

- The geographically distributed nature of platforms and units of a large Organisation (such as a fleet). Direct and seamless communication and knowhow transfer is an efficiency marker.

- The career path of staff requires frequent transfer and reallocation. The new post job description might be completely different and the knowledge acquired is not easily passed on during the traditional transfer of portfolio from the predecessor to the newcomer.

- The early retirement schemes due to the specific character of some professions (such as the navy officer) may deprive the Organisation form critical know how. Institutional and legal obstacles do not allow efficient use of the retired from active service personnel.

It is worth mentioning as an example that, a marine engine shaft failure (together with the knowledge acquired by the staff involved in problem solving - data collection, assessment, repair), remains as a faint trace of information in the memory of the ship, when after 3 years, all members of staff may have been re-allocated.

To cope with the above challenges, NAVMAT develops, promotes and establishes a knowledge based system, a platform for the recording, indexing, comparing, assessing and retrieving upon request every publishable information, history of operations and maintenance, evidence and testimony, for incidents of failure in the defined environment.

The variability of language use when reporting, together with the multi-modal assets attached to such events (measurements, photos, etc.) pose a requirement for intelligent systems, beyond simple keyword-based indexing and retrieval.

The proposed design attempts an interdisciplinary approach by integrating Materials Engineering and Informatics under the Management of Knowledge.

\section{State of the Art}

Knowledge Management (KM) has been linked to artificial intelligence for more than 20 years. Such management has a strong organizational component, beyond the technical requirements. In early works, the challenge for forming a knowledge management culture within an organization was reported to be estimated as " $90 \%$ of the effort" by business leaders [1]. Today, with the increased digitization of business and the transfer of knowledge management in everyday practice, the cultural gaps has been somewhat reduced. However, a number of settings may still retain limited access to effective, user-friendly knowledge management systems. So, regardless of the fact that today we talk about Industry 4.0 and the Internet of Things (IoT) [2] as well as Condition Monitoring and Diagnostic Engineering Management (COMADEM) [3], even reactive (vs. proactive) use of preexisting knowledge at the human expert level for material failures is still an open challenge [4].

In the literature one can find approaches and systems that utilize text understanding in manufacturing [5] to improve "maintenance knowledge intelligence". Through analyses in maintenance reports, operators' workbooks and digital logbooks such systems aim to identify hidden patterns and relations between settings, incidents and human reactions. These approaches, however, may act in a posteriori manner, not facilitating the user in the data entry process as NAVMAT does. 
Expert-system-based approaches have been applied in the past [6] for settings such as onshore pipelines, where again the focus is on the post-entry analysis and organization of documents.

Other approaches focus on the analysis of machine maintenance and use in a cloud setting, to estimate the remaining useful life of components [7]. In such case the aim is to optimize predictors and not to allow for use and re-use of human knowledge as part of a collaborative system.

The development of a knowledge management of automobile system failures [8] was founded of the creation of a failure knowledge ontology from maintenance experience, which allows connecting the pre-existing human understanding of the domain to the knowledge system at hand. In the NAVMAT setting, we utilize a similar view of preexisting knowledge, by initializing a related ontology (in the Lexical and Semantic resources components) which can evolve over time, based on everyday system use.

Case-based reasoning techniques are considered to be the most suitable for generic failure analysis due to the complexity of knowledge required [9]. Such techniques have been employed to support users in hard disk failure analysis [10], uses a reverse-index based approach (Lucene.NET, [11]) and a cosine-similarity metric to identify similar use cases. In our setting, we take advantage of the expert ontology to allow for intelligent and agile search and retrieval, but also to facilitate data entry.

In the medical field, the need for image interpretation (e.g. in bone fractures) has triggered the development of algorithms based on Artificial Neural Network (ANN) architectures [12]. Such systems may involve unsupervised or supervised learning [13, 14].

Thus, NAVMAT aims to provide a point of reference for human-centric, intelligence knowledge management of naval materials failure, throughout the full lifecycle of such information, from knowledge encoding, to incident data gathering and entry, to search and reuse for decision support.

\section{The concept and the tools}

The main scientific challenges in NATMAT reside in (a) knowledge engineering and (b) information retrieval and summarization. Knowledge engineering aims to develop the underlying common understanding of terms, senses and their relations. This, in turn, supports concise definition and storage of incidents. Finally, indexing, retrieval and summarization can be empowered by these definitions and the underlying ontology knowledge to fine-tune incident search and retrieval and to focus on the desired information.

Converging to a common vocabulary and set of relations between terms is critical: such (meta-) information guides storage, indexing and retrieval, touching all main aspects of the system. The ontology encapsulates concepts of the systems, processes but also the security setting, forming a universally understood language within the design.

The process-centric approach in the creation and evolution of the system ontology $[15,16]$ uses ontology engineering patterns where appropriate [17]. Established tools, such as Protégé, are employed to support the editing and evolution of the ontology over time till convergence, providing (a) support for editing ontologies, (b) full change tracking and revision history, (c) collaboration tools and (d) multiple file formats supported for upload and download of ontologies (RDF/XML, Turtle, OWL/XML, OBO, and others).

On the other hand, text mining tools early on (e.g. keyword extraction methods) are utilised, to identify possible terms/keywords and facilitate the ontology creation process dynamically during its evolution.

Automatic text classification is employed [18] to suggest tags to new incidents, facilitating the user and the uptake of the system by experts. To this end, the classification 
takes into account previously labelled incidents as training instances, to form a consistent understanding of existing labels. Over time, newly entered incidents and related work will be provided to the system for more training, creating a virtuous circle of improvement.

Efficient indexing and retrieval (cf. Apache Lucene - SolR / Elastic Search) further enhance the process, allowing the user to leverage the strength of the ontology to get meaningful matches, beyond keyword and boolean search.

\section{System architecture}

NAVMAT builds its architecture taking into account the main business flows. In Figure 1: System overview, we summarize the flow of information, also depicting the main system components (NAVMAT Platform Box):

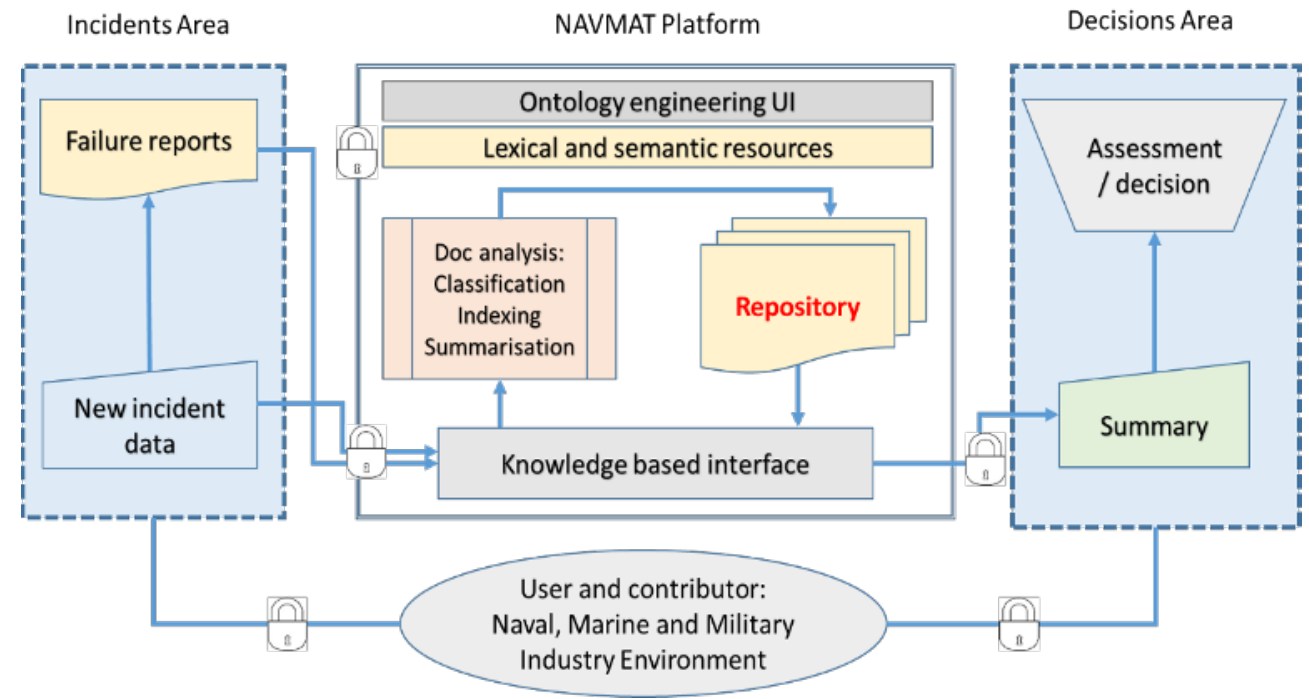

Figure 1: System overview

- Ontology engineering UI: This component is essentially a front-end for domain experts, allowing them to define the main concepts of the domain and their interrelations (Fig.2). This process essentially encodes the domain knowledge in a machineusable format, exploitable by the Document analysis and the Knowledge-based Interface components.

- Lexical and semantic resources: These resources combine the output of the ontology engineering UI with other external resources (e.g. dictionaries or pre-existing ontologies/thesauri) to complement the domain knowledge by encoded real-world knowledge (such as synonyms lists, established encodings of failures, etc. - Fig.2).

- Document analysis: This component is one of the two main components that bring the power of artificial intelligence (AI) into the NAVMAT platform. It allows the automatic annotation of input documents with labels/tags (classification) that facilitate the indexing of the documents and summarization of the related information. The resulting tagged documents are then fed to the repository for later retrieval and re-use, as appropriate.

- Repository: This storage component allows the efficient storage and indexing of documents -and in general resources, e.g. images, audio, text- that constitute the main 
body of information within NAVMAT. These documents are input by the Document analysis component to be later sought and retrieved by the knowledge based interface requests. Published [19-23] and unpublished work of some of the authors will provide the testbed and initial content of the system.

- Knowledge-based interface: This interface allows the users to implement two different workflows:

- Incident data entry: This workflow supports the user when a new incident description is to be added to the system. The component follows the text and documents entered by the user and suggests labels/keywords (provided by the document analysis component) for the efficient indexing of the document. The user can confirm the appropriate labels to finalize the storage.

- Querying previous data: This workflow allows an efficient search of previously stored incidents, by means of an appropriate query. This query can be an incident description in itself or a term/concept-based query. This query results in a number of related pre-entered incidents (from the Repository) which are selected and prioritized to be provided to the user. Essentially this flow also utilizes the summary of information per incident to facilitate the user when skimming through all appropriate incidents to find the most related information. Such information is meant to support the user for the final assessment and/or decision as appropriate.

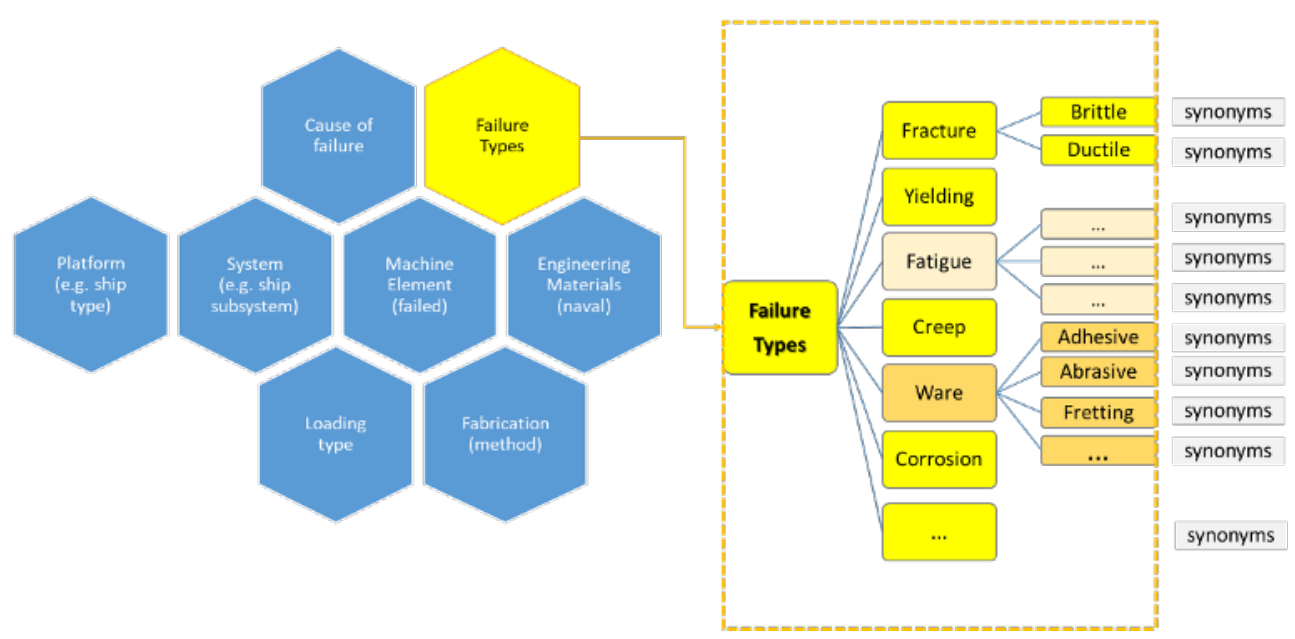

Figure 2: Ontology and taxonomy with the semantic resources for one indicative class (failure types) of the NAVMAT system

\section{Key features}

\subsection{System strengths}

\subsubsection{Multi-linguality}

Protégé [24, 25], a well-established, open-source framework, supporting multi-lingual ontology editing, handles the creation and maintenance of ontologies. Various stakeholders have adopted Protégé as its output can be easily integrated with rule systems or other 
problem solvers to construct a wide range of intelligent systems. Also, in our case, the NAVMAT lexical and semantic resources, a combination of Protégé's output with other possible external resources, supports the analysis and extraction tasks performed by the Document analysis component.

\subsubsection{Scalability}

The NAVMAT platform aims to include various sources of (materials and systems failure) incidents: a) previously stored incidents, b) relevant publications or technical analyses proposed by our experts (the system users responsible for the assessment reports), and/or c) other public failure incidents information and open access publications, identified and suggested by our semantic content analysis and information extraction component.

Additionally, multi-modal assets, such as documents (diagnostics, publications, reports) and media files (photos, videos, audios) can be attached to incidents. Therefore, every single incident of failure can have various sources of supporting documentation.

\subsubsection{Adaptivity / personalization}

- Varying levels of access: the system supports a hierarchy of users, with specific permissions and level of access to information according to their role.

- Learning from user actions: the NAVMAT system intends to enable communication and interaction between its users, as well as to facilitate the management of knowledge and documents related to incidents. As such user interactions and suggestions multiply over time, they will be used as training instances by our expert system, creating a virtuous circle of improvements in user experience.

\subsection{System interface \& security}

The web interface of the NAVMAT system essentially refers to one or more thin clients (Web / mobile app). Indicative workflows include: a) CRUD (create/read/update/delete) operations on reports / incidents / documents. b) Requests suggestions from the Document analysis component, concerning main concepts in the text. c) Enrichment of inserted documents through the Document analysis component. d) Storage of the enriched document into the repository. e) Efficient searching (based on ontology) for previous related incidents and/or related resources (publications, videos, etc.).

Additionally, the system will evaluate the use of well-established practices for sufficient security. This implies, indicatively: a) Use of end-to-end encryption: Protocols such as https will be used to ascertain encrypted, secure communication. b) Secure login mechanisms: Login could be coupled with improved security mechanisms, such as two-factor authentication. c) Data isolation: Use of isolated hosting of data per organization vs. cloud data storage.

\subsection{System potential}

The NAVMAT system has the ambition to integrate the currently existing and fast growing know-how in failure of materials and components with decision making on Maintenance, Repair, Overhaul/Operations (MRO), utilising Informatics and Telecommunication tools. The development of a knowledge base system of such nature, does not concern only the naval materials value chain. The open source of several elements of the system allows a degree of know-how transfer to other industrial activities that may benefit: in the same 
thematic field (other industrial materials failure), similar thematic fields (e.g. forensics) or adjacent thematic fields (failure of electrical/electronic components).

\section{Expected Outcomes}

After the completion of the development, the feeding of the repository with currently available published and unpublished proprietary documents of the partner organisations (beneficiaries), the full operation of the platform for the authorised users, the continuous enrichment of the system with new incidents, assessments and reports, the NAVMAT platform is expected to:

- Erase disruptions in the flow of knowledge (due to the high mobility of personnel and the distribution of knowhow across the beneficiary organisations),

- Improve the management of knowledge of critical component failures,

- Support the decision making in maintenance and materials supply of marine environment operations,

- Train and further educate the technical and scientific personnel of the beneficiary organisations,

- Benefit operational readiness,

- Adopt and explore the potential of semantic web for enriching information and knowledge beyond the beneficiaries (access to public failure incidents information and open access publications).

\section{Bibliography}

[1] Liao, S. H. Knowledge management technologies and applications - literature review from 1995 to 2002. Expert systems with applications, 25(2), 155-164 (2003).

[2] Radanliev, P., De Roure, D., Walton, R., Van Kleek, M., Montalvo, R. M., Santos, O., ... \& Anthi, E. Artificial intelligence and machine learning in dynamic cyber risk analytics at the edge. SN Applied Sciences, 2(11), pp. 1-8 (2020).

[3] Nagaraja Rao, B. K., The Role of Artificial Intelligence (AI) in Condition Monitoring and Diagnostic Engineering Management (COMADEM): A Literature Survey. American Journal of Artificial Intelligence. Vol. 5, No. 1, pp. 17-37 (2021)

[4] Talamo, C., Paganin, G., \& Rota, F. Industry 4.0 for failure information management within Proactive Maintenance. In IOP Conference Series: Earth and Environmental Science (Vol. 296, No. 1, p. 012055). IOP Publishing, (2019, July).

[5] Ansari, F. Cost-based text understanding to improve maintenance knowledge intelligence in manufacturing enterprises. Computers \& Industrial Engineering, 141, 106319 (2020)

[6] Castellanos, V., Albiter, A., Hernández, P., \& Barrera, G. Failure analysis expert system for onshore pipelines. Part-I: Structured database and knowledge acquisition. Expert Systems with Applications, 38(9), 11085-11090, (2011).

[7] Mourtzis, D., Vlachou, E., Milas, N., \& Xanthopoulos, N. A cloud-based approach for maintenance of machine tools and equipment based on shop-floor monitoring. Procedia Cirp, 41, 655-660, (2016).

[8] James, A. T., Gandhi, O. P., \& Deshmukh, S. G.. Knowledge management of automobile system failures through development of failure knowledge ontology from maintenance experience. Journal of Advances in Management Research. (2017) 
[9] P.J. Graham-Jones, B.G Mellor, Eng. Failure Analysis 2 (2), 137-149 (1995)

[10] Wichawong, P., \& Chongstitvatana, P. Knowledge management system for failure analysis in hard disk using case-based reasoning. 18th IEEE/ACIS International Conference on Software Engineering, Artificial Intelligence, Networking and Parallel/Distributed Computing, SNPD, IEEE, pp. 1-6 (2017)

[11] Lucene.NET, https://lucenenet.apache.org

[12] Tang A, Tam R, Cadrin-Chenevert A, Guest W, Chong J, Barfett J, Cheplev L, Cairns R, Mitchell R, Cicero M, Poudrette MG, Jaremko JL, Reinhold C, Gallix B, Gray B, Geis R., Can Assoc Radiol J., 69 120-35 (2018)

[13] Erickson BJ, Korfiatis P, Akkus Z, Kline TL. Radiographics 37(2), 505-15 (2017)

[14] C. Rainey, J. McConnell, C. Hughes, R. Bond, S. McFadden Intelligence-Based Medicine 5100033 (2021)

[15] Zablith, Fouad; Antoniou, Grigoris; d'Aquin, Mathieu; Flouris, Giorgos; Kondylakis, Haridimos and Motta,Enrico. Ontology evolution: a process-centric survey. The Knowledge Engineering Review, 30(1) pp. 45-75 (2015).

[16] Zablith, Fouad (2009). Evolva: a comprehensive approach to ontology evolution. In: 6th European Semantic Web Conference, 31 May - 4 Jun 2009, Heraklion, Crete, Greece, Springer-Verlag, pp.944-948, (ESWC 2009)

[17] Presutti, V., Daga, E., Gangemi, A., \& Blomqvist, E.. eXtreme design with content ontology design patterns. In Proc. Workshop on Ontology Patterns. (2009, October)

[18] Giannakopoulos, G., Mavridi, P., Paliouras, G., Papadakis, G., \& Tserpes, K. Representation models for text classification: a comparative analysis over three web document types. In Proceedings of the 2 nd international conference on web intelligence, mining and semantics (p. 13). ACM (2012).

[19] Karalis, D.G., Melanitis, N.E., Yannoulis, Y.G., Failure analysis of a $\mathrm{Cu}-12 \mathrm{Mn}$ mechanical fastener in marine environment, Eng. Failure Analysis, 94, pp. 69-77 (2018)

[20] Lekatou, A.G., Mpalanou, M., Lentzaris, K., Karantzalis, A.E., Melanitis, N., Microstructure and surface degradation of $\mathrm{Al}$ reinforced by $\mathrm{AlxW}$ intermetallic compounds via different fabrication routes, MATEC Web of Conferences, 188, 03001 (2018)

[21] Karalis, D.G., Melanitis, N.E., Analysis of a premature failure of a hub from a diesel generator of a high-speed motor ship, Journal of Failure Analysis and Prevention, 14(2), pp. 236-246 (2014)

[22] Paipetis, A.S., Aggelis, D.G., Barkoula, N.M., Matikas, T.E., Melanitis, N., Damage monitoring of composite laminates using ultrasonics, Emerging Technologies in NonDestructive Testing V - Proceedings of the 5th Conference on Emerging Technologies in NDT, pp. 281-286 (2012)

[23] Karalis, D.G., Melanitis, N.E., Pantelis, D.I., Failure analysis of a rock anchor made of stainless steel in marine environment, Eng. Failure Analysis, 19(1), pp. 123-130 (2012)

[24] https://protege.stanford.edu/, https://protege.stanford.edu/about.php\#citing

[25] Musen, M.A. The Protégé project: A look back and a look forward. AI Matters. Assoc. of Computing Machinery Specific Interest Group in Artificial Intelligence, 1(4), (2015).

\section{Acknowledgement}

The research project NAVMAT is supported by the Hellenic Foundation for Research and Innovation (H.F.R.I.) under the "1st Call for H.F.R.I. Research Projects to support Faculty Members \& Researchers and the Procurement of High-and the procurement of high-cost research equipment grant" (Project Number: 822). 